\title{
A Web-based Nutritional Assessment Tool
}

\author{
Raluca-Monica POP ${ }^{1}$, Marian POP ${ }^{2}$, Grigore DOGARU ${ }^{3}$, Vladimir C. BACAREA ${ }^{1}$ \\ ${ }^{1}$ Department of Medical Research Methodology, \\ University of Medicine and Pharmacy, \\ 38, Ghe. Marinescu Street, TîrguMureş, 540000, Romania, \\ ralucapetri@yahoo.com \\ 2 Doctoral School \\ University of Medicine and Pharmacy, \\ 38, Ghe. Marinescu Street, TîrguMureş, 540000, Romania, \\ ${ }^{3}$ Department of Internal Medicine \\ University of Medicine and Pharmacy, \\ 38, Ghe. Marinescu Street, TîrguMureş, 540000, Romania,
}

\begin{abstract}
The aim of this study was to present a web-based nutritional assessment tool that offers the possibility to construct the personal food pyramid and interprets the metabolic profile of a patient. A web application was developed both for healthcare professionals and the general public that besides analyzing the eating habits also provides some specific recommendations for every user. After filling in some general information the user has the choice either to go to the interpretation or to complete a food frequency questionnaire that allows a thorough analysis of his/hers eating pattern. The advantages of this tool are its accessibility, the construction of the personal food pyramid and the fact that is developed in a home country.
\end{abstract}

Keywords: food pyramid, web-based tool, nutrition.

\section{Introduction}

The obesity epidemic has become a reality and a great number of interventional studies have been developed for its prevention and treatment, given the immense financial burden swallowed by this disease. Nutritional assessment tools are available throughout the world-wide-web more or less controlled or based on guides or professional information. The last decades have witnessed a great increase in the number of tools that help health professionals in their work. Although the nutritional assessment should be a part of the general evaluation, it's time consuming and usually it resumes to a few general questions. Western economies have been using different types of assessment tools for developing national guidelines and recommendations [1, 2, 3].

The wide spread of computer users has switched the interest towards constructing new, easy to use web applications that can allow a more user-friendly approach to a difficult problem. The involvement of the patient in his/hers evaluation has many advantages, but, at the same time, there are flaws that cannot be controlled.

Food frequency questionnaires are a valuable method of nutritional assessment, using a list of foods and some options concerning the frequency of consumption of the foods listed. They are usually designed to gather information from large populations and are usually self-administered. Their strengths consist in: low respondent burden, suitable for large scale surveys, can be self-completed, can be posted. They also have some disadvantages: the need for estimation of portion size, possibly over-reporting of "healthy foods" [4].

\section{Material and Method}

A web application was developed for nutritional assessment, using Apache for web server and MySQL as a database management system. This was designed to be used for patients' management in medical office, both for general practitioners and registered nutritionists once it has been deployed on internal or web-based sites.

Back-end coding was made in PHP 5. Frontend coding was made using Twitter Bootstrap, a free popular front-end framework. Also jQuery was used as the main Javascript library. Using this framework we created the graphical user interface (GUI). After the compilation, the framework creates a single HTML file and a JS file accessed on page load. One of the advantages using Twitter Boostrap framework is that the GUI performs and looks the same on most of the popular browsers and operating systems (OS). 
Communication with the database layer is done using the php back-end which executes all queries, synchronous and asynchronous, sent from the GUI. The received information is displayed without accessing a different URL, given that the application runs on the page. The application can be accessed using any of the web browsers available, with Javascript turned on.

Data storage - The patient data is stored into a database consisting of 8 tables (34 fields). We found MySQL to be suitable for this kind of application, given the need for a relational database and a fast response time [5].

The food frequency questionnaire (FFQ) used has been developed based on the National Health and Nutrition Examination Survey (NHANES) and adapted to local foods with the aid of a registered endocrinologist. A written form of the FFQ is already in use and has showed fairly good results in nutritional evaluation [6].

The answers obtained in the FFQ about the number of times each food was consumed are transformed in number of times they're consumed/day. Each item belongs to one or more of the major food groups. In the end based on this algorithm, the applications constructs the food pyramid with the 6 food groups and the number of servings/day.

During the development phase a pilot study was conducted to evaluate the GUI and the users' level of acceptance. Students from our university were invited to use the tool. A sample of 37 students completed the questionnaire in an average time of 15 minutes. They could offer their suggestions and opinions at the end. They agreed that the application was fairly easy to use, with comprehensive explanations at the end.

\section{Results}

The Graphical User interface consists in a few pages:

1. authentication page (e-mail)

2. user general datapage

3. intermediary page with general information about the quiz

4. edit/continue quiz

5. quiz results and patient diagnostics based on patient data.
1. On the authentication page the user introduces his/hers e-mail and password provided by the administrator in order to proceed to the next page (Figure 1).

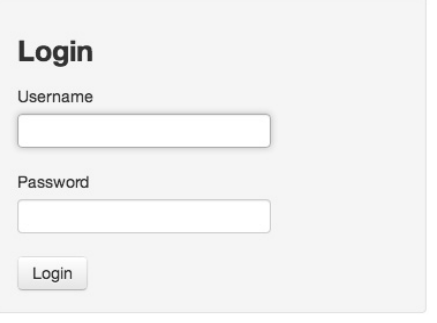

Figure 1. Authentication

2. On the user generalpage, the user fills some mandatory information-age, sex, environment, height, weight, waist circumference. There are also some other optional fields that can be filled: maximum systolic blood pressure, total cholesterol, HDL-cholesterol, triglycerides, blood glucose, and serum creatinine. For the first 4 there's also the option of checking a box if the patient is undergoing treatment for these variables. There are also 2 boxes that cannot be modified: the BMI (body mass index) and the eGFR (estimated glomerular filtration rate) that will be shown on the result page, after calculation based on formulas integrated in the application. (Figure 2)

The form provides three actions:

- Cancel

- Save - saves the information and returns to patient list

- Save and Continue with Quiz - saves the information and redirects to general information about the quiz.

3. The intermediary page provides general information about the quiz and presents the rules for filling the questionnaire. It also explains what the portion definition for each type of food is.

4. The questionnaire. For each item, the user must choose one of 10 possibilities of answers, ranging between never and more than 6 servings per day, for each type of food presented. There are 126 questions in the questionnaire, divided in the 6 main food groups. For each item the user can see the food group to which it is associated. The progress of the quiz is also presented. Every answer is automatically saved in the database - after choosing one of the ten options, the application shows the next question. The user can choose 


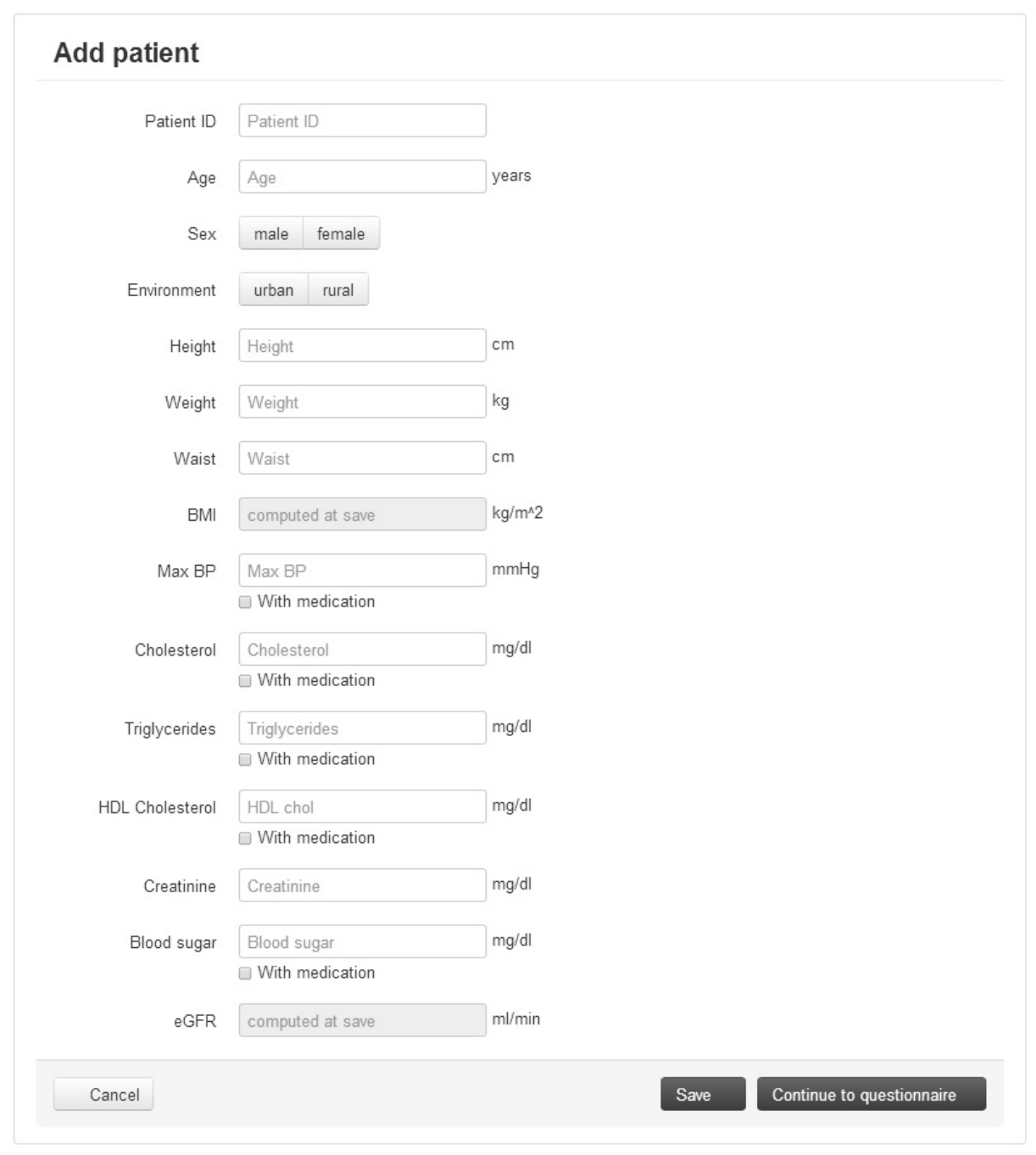

Figure 2. The add/edit patient page

to finish the quiz or to save the progress and return later to finish it. Also, the answers can be modified at any time during the quiz. (Figure 3 )

5. The results page has two main parts: results based on interpretation of the general information filled by the user on the add/edit patient page and the questionnaire interpretation. The user has the option of printing this result page. If the quiz is not completed, there will be no quiz results.

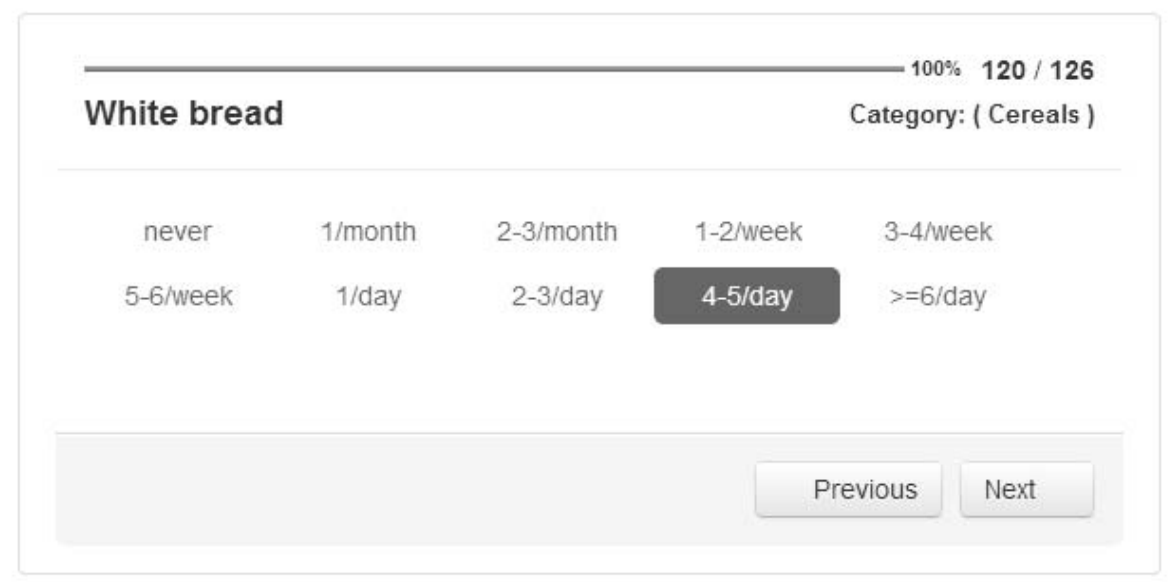

Figure 3. An example of an item in the questionnaire 
The first part of this results page offers information about the weight, waist circumference, lab values. For values within the normal range we used green colored boxes and for those outside the normal range, red boxes. This color code is used also for the quiz results. For the values outside the normal range there's also a message with recommendations for improvement, or medical consult.

The normal range for each variable is based on generally accepted formulas or international guidelines [7, 8].
For the body mass index the application also calculates the normal weight range based on age, gender and height and the amount of weight gain/loss needed to reach the normal (Figure 4).

The second part of the results page offers the questionnaire results. It consists of two parts: the quiz analysis and general recommendations. After the questionnaire is completed, the application constructs the individual food pyramid and it calculates the number of servings/day for each food group. The page

Anthropometry interpretation

\begin{tabular}{lr}
\hline Patient data: \#143/13.05 & Print results \\
\hline Age & 64 years \\
\hline Sex & feminin \\
\hline Environment & urban \\
\hline Height & $152 \mathrm{~cm}$ \\
\hline Weight & $72 \mathrm{~kg}$ \\
\hline Waist & $93 \mathrm{~cm}$ \\
\hline & \\
\hline Your waist exceeds the recommended upper limit. Your maximum target waist should be $80 \mathrm{~cm}$. \\
\hline BMI & $31.16 \mathrm{~kg} / \mathrm{m}^{\wedge} 2$ \\
\hline
\end{tabular}

Blood variables interpretation

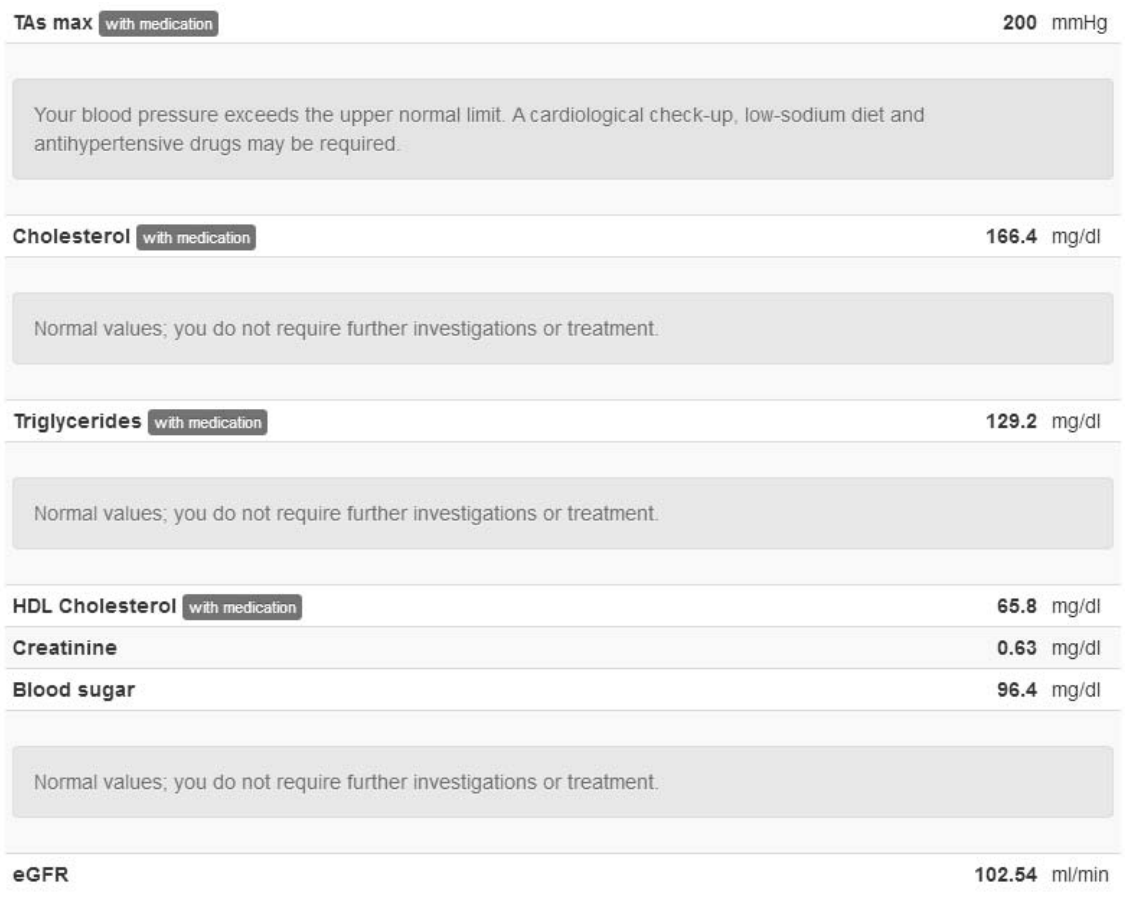

Figure 4. General results 
displays the normal food pyramid and the one obtained based on the answers. Next, it shows the basal metabolic rate calculated using the Harris Benedict equation [9]. For each food group there's an analyzing box - red for the foods consumed in higher/lower amounts than the recommended number of servings and green for those consumed in the right amount. The last part of the results shows general recommendations for a healthy diet, finishing with an example of a healthy plate (Figure 5).

All the recommendations are based on nutritional guidelines and written by a registered

Personal food pyramid

Patient data: \#125/24.04

Questionnaire results

\begin{tabular}{|c|c|c|c|c|c|}
\hline \multicolumn{2}{|l|}{ Food groups } & \multicolumn{4}{|c|}{ Food pyramid } \\
\hline Group & Servings/day & $\mathrm{Nr}$ & Normal & $\mathrm{Nr}$ & Your pyramid \\
\hline Sweets & 0.2 & 1 & $\square$ & 0.2 & ! \\
\hline Fat & 0.96 & 2 & & 0.96 & L \\
\hline Meat \& Protein & 0.94 & 2 & & 0.94 & $\square$ \\
\hline Dairy & 1.86 & 3 & & 1.86 & $=$ \\
\hline Fruits \& Vegetables & 6.96 & $5+$ & & 6.96 & \\
\hline Cereals & 2.5 & $6+$ & & 2.5 & \\
\hline
\end{tabular}

Basal Metabolic Rate

1151.6 calories

*required for "being alive"

Food groups Interpretation

Sweets

You consume sweets within the recommended quantity. This food group is not essential for your health and must

not represent an important source of calories.

Fat

You do not eat the recommended quantity of fat Choose mostly the vegetable fats seeds, nuts, avocado, olive oil. Fats are necessary for the absorption of liposoluble vitamins which are essential for health: vitamins A, D, E, K.

Meat \& Protein

You do not eat enough meat and protein. White meat is recommended - fish or poultry, skim. Eggs are also

source of proteins. So are beans and peas. For example you can choose $100 \mathrm{gr}$ grilled chicken breast and a boiled egg and you reach the recommended number of meat servings/day.

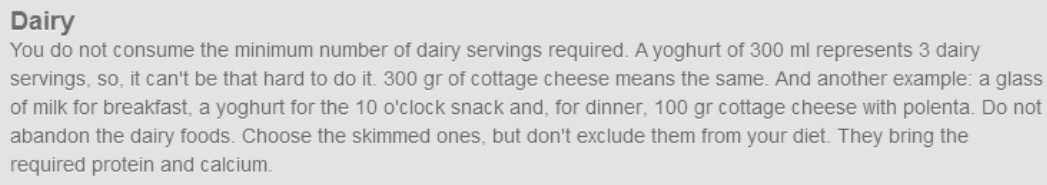

Fruits \& Vegetables

You consume the right amount of fruits and vegetables. Don't forget to choose as often as you can the vegetables over fruits, because the latter are rich in sugar which is not recommended but in minimum amounts.

\section{Cereals}

You do not consume the minimum number of cereal servings/day. Don't forget that a serving is equal to a slice of

bread (not that homemade which is already at least 2 servings), a biscuit, a bagel, $100 \mathrm{gr}$ of cereals, a medium

potato

Figure 5. Quiz interpretation 
endocrinologist with focus on obesity.

The user is only able to view his/hers results, while the administrator can see the entire database, can add/delete any information and is the only one allowed to add authentication information.

\section{Discussions}

The obesity epidemic has become a great health issue. Giving the lack of treatment options and the fact that the great majority of obese people have bad diet habits, the nutritional assessment is one of the most important parts of a patient evaluation. Although there's a general agreement for its importance, it is many times overlooked, because it is time consuming and difficult to accomplish. The wide spread of computer users throughout the world has given the opportunity to develop new methods for the nutritional assessment. In the past years, a great number of applications have become available, many of them not having the necessary professional guidance underneath. Also, some of them are not available for free and the great majority is only available in foreign languages [10]. There is consensus among nutritionist that web-based interventions and evaluations are a useful tool, which will become more and more important in future years [11]. Considering these problems, we developed an easy to use web-based application that does not require former knowledge of the nutritional assessment methods, or an important previous computer knowledge/experience.

As others authors stated [12], using a web platform has many advantages. It's available on any web browsers, the computer's resources are minimally used, information is secure, easy to backup and the maintenance is not difficult. Given the fact that it uses a web platform, it can be accessed anywhere, by multiple users, with the sole condition of having a solid internet connection.

Similar applications have been developed in different languages and the conclusion was that these tools are very easy to use, do not require great computer management knowledge and are very useful both for surveys and personal evaluations [13, 14].

The constructed pattern allows future development, with the possibility of adding more nutritional assessment tools, a list of foods, more formulas, etc.

One of the disadvantages of this tool is that is a little bit time consuming, given the fact that the questionnaire consists of 126 items. To resolve this problem, the application gives the opportunity of filling the quiz at different times, with the sole condition that the interpretation is only generated when all the questions have been answered. We chose the food frequency questionnaire as a nutritional assessment tool because is the method that allows obtaining a wide perspective on a patient eating habits [15]. The questionnaire was developed based on the one used by the National Health Institute in the survey that underwent in 2008, adding some specific Romanian foods and eliminating those not so often used [2]. We cannot pretend that the food list is flawless, but it has the usual number of items needed to give a general image on a patient's diet habits, it's well adapted to the local food customs and it has at least 10 items for each food group.

The interpretation and recommendations for the variables analyzed are based on international guidelines and can be verified at any given time $[16,7,17,18]$ so we can state that they are reliable. We must underline that the interpretation is age specific, using the BMI, waist circumference, blood pressure percentiles and also the cut-off levels for children regarding the lab results.

The printable report at the end of the evaluation gives the patient a very good image on his/hers nutritional status, also providing recommendations for improvement. These recommendations are based on national and international nutritional guidelines and are compiled by a registered endocrinologist with focus on obesity and nutrition [18, 3]. Also, another advantage of using this web-platform is the construction of the personal food pyramid. Each item belongs to a food group (some of them to 2 or more) and each answer is added up to calculate the frequency/day.

We found the visual and numerical result to be very helpful in that is allowing a simpler explanation to the patient with categorical recommendations; also an experienced endocrinologist with a focus on obesity treatment found that printable results allowed more tailored recommendations. 
Although we found this web-platform to be very useful, one must take into consideration the users' degree of acceptance. We consider that the target population is represented by people who are using the World Wide Web, but, at the same time, any person consulting an endocrinologist or nutritionist can be explained and can follow the steps to reach the interpretation phase.

Although the web platform assures a fairly good nutritional assessment, for specific cases, a medical consult is recommended.

Considering the issue of privacy, we can say it's fairly assured by the encrypted password, the fact that one user is allowed to see only his /hers page and the fact that the administrator, a medical practitioner is bound by oath to keep the information about patients secure and private.

Other applications developed in Romanian [19] only provide a list of food items and the nutrient composition, without a thorough assessment. As future perspective, we think this web-platform can be modified in becoming a true nutritional tool, allowing both professional and personal usage.

\section{Conclusions}

The application we're proposing allows an elaborate nutritional assessment, based on pertinent recommendations and guidelines. It is easy to use and has the advantage of being developed in our native language. It is the only available application that constructs the individual food pyramid. Its form allows future development with integration of a physical activity tracker, a 24 hour dietary recall, a food list. We consider this web-platform useful both for doctors and the general population, raising health awareness.

\section{Acknowledgements}

This paper is partly supported by the Sectorial Operational Program Human Resources Development (SOP HRD), financed from the European Social Fund and by the Romanian Government under the contract number POSDRU 80641. Project partly supported through an Internal Research Grant of University of Medicine and Pharmacy TirguMures, 13/30.01.2013.

\section{REFERENCES}

1. WRIEDEN, W., H. PEACE, J. ARMSTRONG, K. BARTON. A Short Review of Dietary Assessment Methods used in National and Scottish Research Studies. [Internet]. 2003

2. National Cancer Institute. Risk Factor Monitoring and Methods, [Internet]. 2008.

3. U.S. Department of Agriculture and U.S. Department of Health and Human Services. Dietary Guidelines for Americans. 7th ed. Washington D.C.: U.S. Government Printing Ofice; 2010.

4. THOMPSON, F. E., A. F. SUBAR. Dietary Assessment Methodology. In: Ann M. Coulston CLRaERM. Nutrition in the Prevention and Treatment of Disease. Elsevier; 2001. pp. 3-30.

5. MUJI, M., R. V. CIUPA, D. DOBRU, C. BICA, P. OLAH, V. BACAREA, M. MARUSTERI, Database Design Patterns for Healthcare Information Systems. In: IFMBE Proceedigns, Cluj Napoca, 2009, pp. 63-66.

6. POP, R., M. POP, G. DOGARU, Assessing the Patient with Abdominal Obesity: Metabolic and Nutritional Factors. In: The 6th Conference of PhD Students, Book of Abstracts, Tirgu Mures, 2013, p. 32.

7. World Health Organization. World Health Organization. [Internet]. 2013.

8. COCKCROFT, D. W., M. H. GAULT, Prediction of Creatinine Clearance from Serum Creatinine. Nephron. 1976, vol. 16, pp. 31-41.

9. HARRIS, J. A., F. G. BENEDICT. A Biometric Study of Basal Metabolism in Man. Washington: Carnegie Institution op Washington; 1919.

10. United States Department of Agriculture. Choose my plate. [Internet]. 2012 .

11. BRUG, J., A. OENEMA, M. CAMPBELL, Past, Present, and Future of Computertailored Nutrition Education. American Journal of Clinical Nutrition vol. 77, 2003, pp. 1028-34.

12. GHIGA, D., M. PETRISOR, A. BACAREA, V. BACAREA, M. MARUSTERI, B. HAIFA, D. 
SIMPALEAN, A. SCHIOPU, A Computer-based Decision Support System Used in Identifying Relationship between Oral Symptoms and Systemic Diseases. Studies in Informatics and Control, vol. 21, 2012, pp. 267-74.

13. OENEMA, A., J., BRUG, L. LECHNER, Web-based Tailored Nutrition Education: Results of a Randomized Controlled Trial, Health Education Research, vol. 16(6), 2001, pp. 647-60.

14. GONZÁLEZ CARRASCOSA, R., J. L. BAYO MONTÓ, T. MENEU BARREIRA, P. GARCÍA SEGOVIA, J. MARTÍNEZMONZÓ, Design of a Self-administered Online Food Frequency Questionnaire (FFQ) to assess Dietary Intake among University Population. Nutrition Hospital, vol. 26(6), 2011, pp. 1440-6.

15. SUBAR, A. F., K. W. DODD, P. M. GUENTHER, V. KIPNIS, D. MIDTHUNE, M. MCDOWELL, J. A. TOOZE, L. S. FREEDMAN, S. M. KREBS-SMITH, The Food Propensity
Questionnaire: Concept, Development, and Validation for Use as a Covariate in a Model to Estimate Usual Food Intake. Journal of American Diet Association, vol 106, 2006, pp. 1556-63.

16. American Association of Clinical Endocrinologists. Guidelines for Management of Dyslipidemia and Prevention of Atherosclerosis. Endocrinology Practice, vol. 18 (Suppl 1), 2012, pp. 1-78.

17. American Diabetes Association. Standards of Medical Care in Diabetes-2013. Diabetes Care, vol. 36 (Suppl. 1), 2013, pp. 11-66.

18. GRAUR, M., Ghid pentru Alimentatia Sanatoasa. Iasi: Ed. Performantica; 2006.

19. IANCU, S. Ş., C. I. BONDOR, Ş. ŢIGAN, NUTRIBASE - Data Base for Nutritional Evaluation and Dietetic Treatment in Populational Metabolic Diseases, Applied Medical Informatics, vol. 23, 2008, pp. 43-50. 